\title{
Control of tunable, monoenergetic laser-plasma-accelerated electron beams using a shock-induced density downramp injector
}

\author{
K. K. Swanson, ${ }^{1,2}$ H.-E. Tsai, ${ }^{1}$ S. K. Barber, ${ }^{1}$ R. Lehe, ${ }^{1}$ H.-S. Mao, ${ }^{1}$ S. Steinke, ${ }^{1}$ J. van Tilborg, ${ }^{1}$ \\ K. Nakamura, ${ }^{1}$ C. G. R. Geddes, ${ }^{1}$ C. B. Schroeder, ${ }^{1}$ E. Esarey, ${ }^{1}$ and W. P. Leemans ${ }^{1,2}$ \\ ${ }^{1}$ Lawrence Berkeley National Laboratory, 1 Cyclotron Rd, Berkeley California 94720, USA \\ ${ }^{2}$ University of California - Berkeley, Berkeley California 94720, USA
}

(Received 1 November 2016; published 30 May 2017)

\begin{abstract}
Control of the properties of laser-plasma-accelerated electron beams that were injected along a shockinduced density downramp through precision tailoring of the density profile was demonstrated using a $1.8 \mathrm{~J}, 45 \mathrm{fs}$ laser interacting with a mm-scale gas jet. The effects on the beam spatial profile, steering, and absolute energy spread of the density region before the shock and tilt of the shock were investigated experimentally and with particle-in-cell simulations. By adjusting these density parameters, the electron beam quality was controlled and improved while the energy (30-180 MeV) and energy spread (2-11 MeV) were independently tuned. Simple models that are in good agreement with the experimental results are proposed to explain these relationships, advancing the understanding of downramp injection. This technique allows for high-quality electron beams with percent-level energy spread to be tailored based on the application.
\end{abstract}

DOI: 10.1103/PhysRevAccelBeams.20.051301

\section{INTRODUCTION}

Laser plasma accelerators (LPAs) can produce ultrashort electron bunches at relativistic energies in a short distance [1-5]. This compact source can provide electrons for various applications such as the production of $\mathrm{x}$-ray pulses from coherent undulator radiation or Thomson backscattering [6-9] which can be used for imaging the structural dynamics of chemical and biological systems, highresolution lithography or characterization of nuclear materials [10-12]. However, these applications necessitate stable, high-quality, and tunable electron beams.

The electron beam quality depends on the method used to inject electrons into the plasma's accelerating field and the acceleration dynamics. Large energy spread beams are produced when electrons are injected over an extended distance [13-16]. Energy spread can be reduced by operating near injection threshold or terminating the acceleration when electrons start to outrun the laser, but these methods limit the tunability and repeatability of the LPA $[17,18]$. Using multiple laser pulses allows for more control over the injection process but increases the alignment complexity $[19,20]$.

A tailored plasma density profile can decrease energy spread by reducing the injection length and controlling the injection dynamics [21-25]. In this scheme, an intense laser

Published by the American Physical Society under the terms of the Creative Commons Attribution 4.0 International license. Further distribution of this work must maintain attribution to the author(s) and the published article's title, journal citation, and DOI. pulse propagates through a density downramp from a peak plasma electron density $n_{1}$ to a plateau plasma electron density $n_{2}$. Along the downramp, the plasma wavelength $\lambda_{p}(\mu \mathrm{m})=3.3 \times 10^{10} / \sqrt{n\left(\mathrm{~cm}^{-3}\right)}$ increases with propagation distance. This increase in $\lambda_{p}$ causes the local wake phase velocity to decrease, reducing the threshold for trapping relative to a uniform plasma. This injection process is controlled and localized by the downramp, producing small energy spread beams.

While this scheme has been studied experimentally and quasimonoenergetic beams were produced [21,22,24-26], little investigation has been done to tune the electron beam spatial quality and absolute energy spread by tailoring the density profile. In this contribution, we advance the understanding of injection along a density downramp and demonstrate novel techniques for controlling the electron beam quality. Using a shock-induced downramp [24-26], we determine the relationships between the density profile-including the shock front angle and density upramp region, which to our knowledge have not been examined-with beam parameters such as energy, charge, steering, ellipticity, and energy spread. By adjusting the density profile and tilt of the shock front, stable beams with tunable energy, improved spatial quality and reduced energy spread were produced, demonstrating that this injection mechanism is reliable and versatile enough to be used for many applications.

\section{EXPERIMENT}

The experiments were performed with the BELLA Center's TREX Ti:Sapphire laser, providing pulses with 
$805 \mathrm{~nm}$ central wavelength, 47 fs FWHM pulse duration, and $1.8 \mathrm{~J}$ on target energy. The pulses were focused with a $2 \mathrm{~m}$ off-axis parabolic mirror to an 18 um FWHM beam waist, corresponding to a peak intensity of $4 \times 10^{18} \mathrm{~W} / \mathrm{cm}^{2}$ (normalized vector potential $a_{0}=1.4$ ).

The setup is shown in Fig. 1(a). The target was a supersonic conical hydrogen gas jet with an $840 \mu$ m exit diameter and Mach number $M=2$. A razor blade was placed in the gas flow to produce a shock front that served as a density transition. The blade could be independently positioned through the gas flow. The jet-blade assembly could be rotated to change the shock front angle $\alpha$ with respect to the laser propagation axis and was set to have a $28^{\circ}$ tilt to produce a shock front with $\alpha=0$ when the blade tip was slightly past the center of the jet.

The electron beam energy spectrum was measured using a magnetic spectrometer. A removable scintillating screen imaged to a CCD camera was used to measure the electron beam profile and pointing. The plasma density profile was characterized in situ using a second laser pulse transverse to the main pulse such that the shock front was imaged onto a wavefront sensor with a $3 \mathrm{~mm}$ field of view and a $30 \mu \mathrm{m}$ imaging resolution [27]. A representative wavefront image is displayed in the inset of Fig. 1(c).

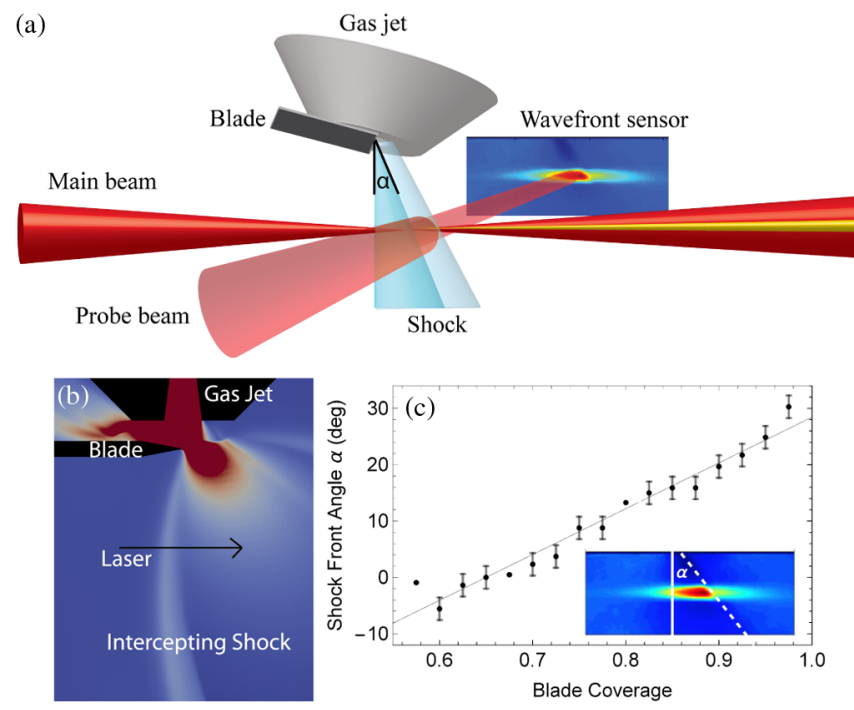

FIG. 1. (a) Schematic of the experimental setup. A razor blade was inserted into the flow of a supersonic gas jet, forming a shock. The main laser beam was focused into the shock region, producing electrons. A probe beam perpendicular to the shock front was imaged onto a wavefront sensor for density measurements. (b) OpenFOAM simulation of the razor blade in the gas flow showing an intercepting shock front. The color scale has been saturated to better see the intercepting shock. (c) Shock front angle versus blade coverage. The inset shows an example phase image from the wavefront sensor where the shock front angle $\alpha$ has been defined. The blue region is neutral density gas and the $\mathrm{red} /$ green is laser-ionized plasma. In both the neutral gas and plasma, the shock front can be seen.
Two-dimensional simulations were performed using a compressible fluid dynamics solver in OpenFOAM to provide an understanding of the shock formation and density downramp [28]. These simulations showed that after the gas reaches the blade, it expands and recompresses into an intercepting shock as shown in Fig. 1(b). The direction of the expanding flow depends on the fraction of the gas jet covered by the blade, or blade coverage, which leads to a blade position-dependent shock front angle [29]. This trend was experimentally confirmed as shown in Fig. 1(c). The shock front angle, $\alpha$, changed linearly with the blade coverage from $\alpha=-10^{\circ}$ to $30^{\circ}$.

Lineouts taken along the laser propagation axis of Abelinverted wavefront images provided density distributions for the plasma regions outside of the shock for each blade location. Because Abel inversions require rotational symmetry, which a titled shock front violates, two subregions of the wavefront images as shown in the inset of Fig. 2(a) were taken. The plasma in each of these regions forms a half cylinder which can be Abel inverted. Density profiles were obtained by combining the two Abel-inverted images and taking a lineout across the center. The shock downramp length cannot be derived from the Abel inversion and was therefore measured from the wavefront images. Displayed in Fig. 2(a) is an example measured plasma density lineout indicating the peak density $n_{1}$, plateau density $n_{2}$, high density length $L_{\text {high }}$ which controls the injection performance, and plateau density length $L_{\text {acc }}$ over which the electrons are accelerated. Stacked density lineouts for various blade coverages are shown in Fig. 2(b). It can be seen that the location of the shock moves with the blade: as the blade moves further into the gas, the shock front moves with it, reducing $L_{\text {acc }}$. The plateau density length $L_{\text {acc }}$ can be tuned up to $2 \mathrm{~mm}$. The density downramp width, defined as the distance between the peak density and the plateau density, was constant at $100 \mu \mathrm{m}$ over the blade coverage range.

It can also be seen in Fig. 2(b) that the densities $n_{1}$ and $n_{2}$ are both functions of the blade position. The plateau density $n_{2}$ varies from 0.8 to $3.5 \times 10^{18} \mathrm{~cm}^{-3}$ while the peak density $n_{1}$ varies from 2.7 to $8.7 \times 10^{18} \mathrm{~cm}^{-3}$ as the blade coverage changes from 0.25 to 1.0 . Measured $L_{\text {acc }}, n_{1}$ and $n_{2}$ values in the range of blade coverages used to produce electron beams are shown in Fig. 2(c).

For each blade position the shock front location was different and therefore, when producing electrons, the laser focal location was adjusted to keep the laser focused onto the shock region. This optimization resulted in the smallest divergence, most stable electron beams for each blade position. Electron beams were produced every shot with down to $0.3 \mathrm{mrad}$ RMS pointing fluctuation, an average of $2 \mathrm{pC}$ charge fluctuation and $6 \%$ energy fluctuation. Figure 3(a) shows the energy spectra for 50 consecutive shots for a blade coverage of 0.8 to demonstrate the stability of this technique. 

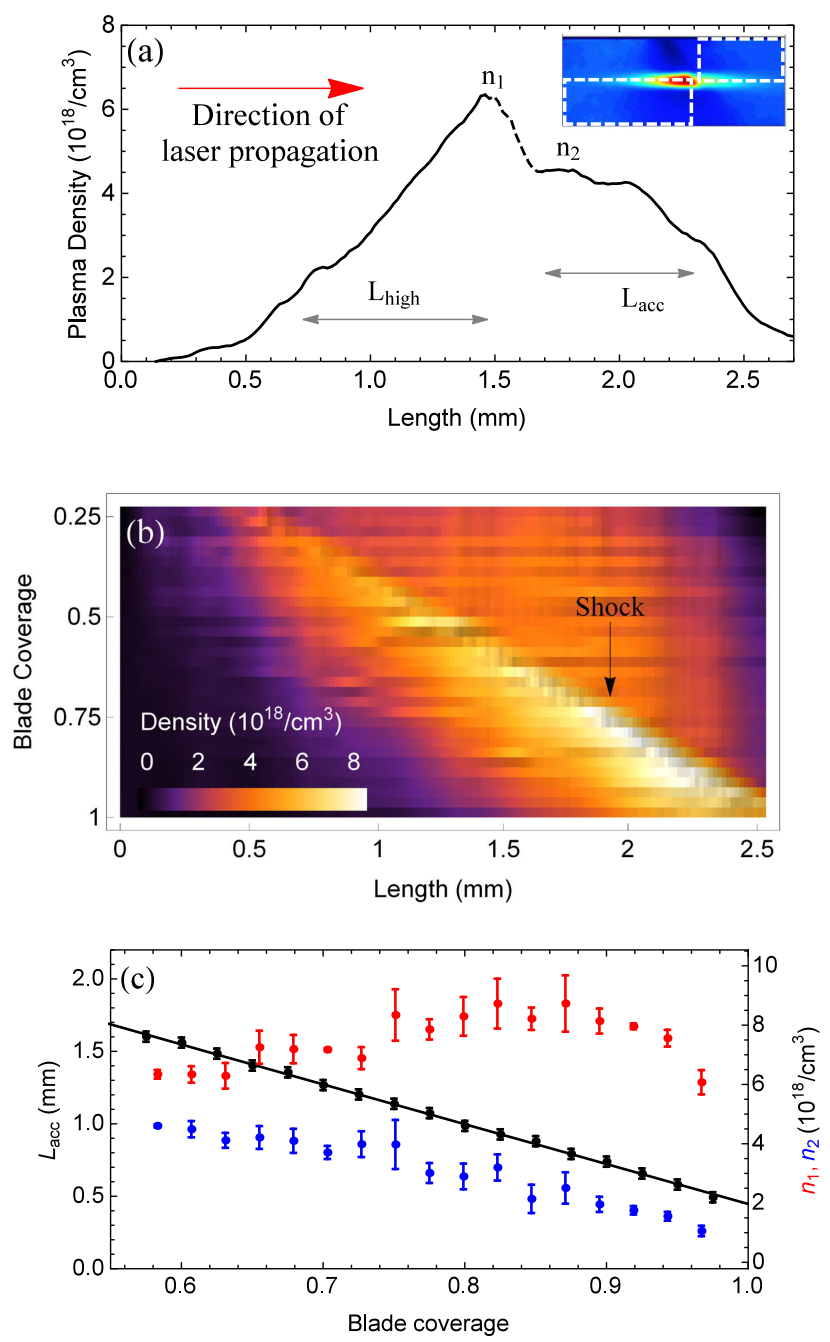

FIG. 2. (a) Plasma electron density profile measured along the laser propagation axis for blade coverage 0.575 . The densities $n_{1}$ and $n_{2}$, the acceleration length $L_{\text {acc }}$, and high density length $L_{\text {high }}$ have been labeled. The dashed line shows the region that cannot be Abel inverted. Pre-Abel inverted wavefront images were used to measure the length of the downramp. The imaging resolution of the wavefront sensor is $35 \mathrm{um}$. Inset shows the two regions for which the Abel inversion was performed. (b) Density lineouts as the blade was translated through the gas jet. Each horizontal line corresponds to a density lineout at a particular blade coverage. Blade coverage of 1 corresponds to the blade completely covering the jet exit. (c) Peak density $n_{1}$ (red), plateau density $n_{2}$ (blue), and plateau density length $L_{\text {acc }}$ (black) for the blade coverages used to produce electron beams.

By changing the blade location, the electron energy was tuned from 32 to $180 \mathrm{MeV}$, as shown in Fig. 3(b). The experimental energy gain, $W$, can be compared with the gain calculated from the simple model $W \approx e E_{z} L_{\text {acc }} \propto$ $\int_{0}^{L_{\text {acc }}} \sqrt{n_{2}(z)} d z$ where $E_{z} \propto 1 / \lambda_{p}$ [2], $L_{\text {acc }}$ is defined as the distance it takes $n_{2}$ to decrease to $\frac{1}{4} n_{2}$, and both $L_{\text {acc }}$ and $n_{2}$ are functions of the blade coverage. At the densities used in this experiment, the accelerator is not limited by laser
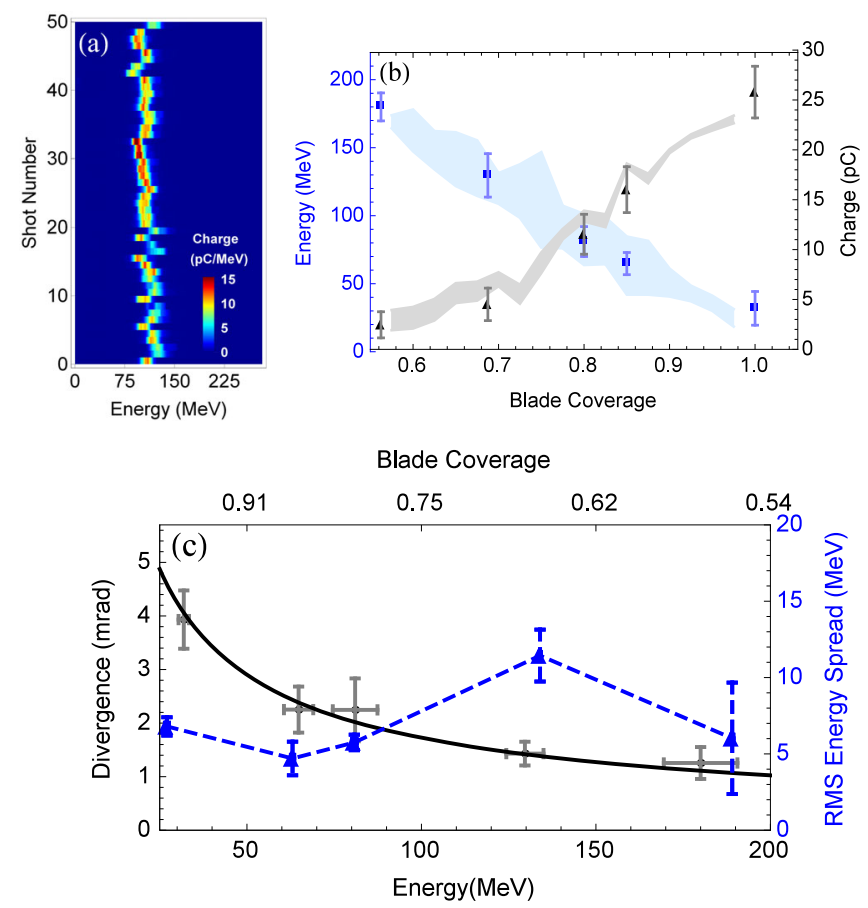

FIG. 3. (a) Energy spectra for 50 consecutive shots when blade coverage is 0.8 . (b) Mean energy (blue squares) and charge (gray triangles) of the electron beams versus blade coverage. Experimental results are displayed as points and values calculated from measured density profiles are displayed as shaded regions. (c) Divergence (black) and absolute energy spread (blue) versus energy. The experimental values are displayed as points and the $\gamma^{-3 / 4}$ fit as solid line. Note the stable absolute energy spread throughout the range of energies. All error bars represent one standard deviation of uncertainty.

depletion, and the electrons can be accelerated over the whole $L_{\text {acc }}$ [2]. Using this scaling law, we fit the calculated electron beams' energies based off measured $n_{2}$ and $L_{\text {acc }}$ to the experimental values, and these fitted energies are shown in the blue area where the width corresponds to measured density fluctuations. The fluctuations in energy, as well as other acceleration parameters, can be attributed to variability in the properties of the target, i.e., gas jet current, or the laser, i.e., energy. Earlier experiments have proven correlations between these parameters and the injected energy; therefore, energy fluctuation can be improved with better gas jet and laser stability. The experimental electron energies are in good agreement with this trend, demonstrating the tunability of this injection technique.

The total injected charge is proportional to the plasma density $n_{1}$ and the injection volume $w_{0}^{2}\left(\Delta \lambda_{p}\right)$ where $\Delta \lambda_{p}=$ $\lambda_{p, 2}-\lambda_{p, 1}$ and $w_{0}$ is the laser beam waist at the shock. Simulations, discussed below, showed that $w_{0}$ weakly depends on blade position and was therefore assumed constant. The fit of $n_{1} \Delta \lambda_{p}$, using measured $n_{1}$ and $n_{2}$ values, to the experimental data is shown in the gray area in Fig. 3(b) and corresponds well with the experimental data. The wake is not strongly suppressed due to beam loading as 
the amount of charge required to beam load is $2 \mathrm{nC}$ [2], which is well above the measured charges.

The beam divergence depends on beam energy with higher energy beams having lower divergence. For each blade position shown in Fig. 3(b), the beam divergence was measured and related to the beam energy. Figure 3(c) shows the FWHM divergence averaged in the vertical and horizontal directions as a function of the energy. In a simplified model of the electron motion in an accelerating field with a linear focusing force and constant normalized emittance, the beam divergence scales as $\gamma^{-3 / 4}$ where $\gamma$ is the electron Lorentz factor [30]. This scaling agrees well with the data as shown by the fit in Fig. 3(c). If the electron beam size is mismatched to the plasma wave, the divergence will oscillate about this $\gamma^{-3 / 4}$ trend. Therefore, within the errors, the injected electrons appear to undergo matched propagation.

For any given blade position, the shock front angle, $\alpha$, may not be perpendicular to the laser. As the laser propagates through the gas, it is refracted due to the overall shape of the gas profile and the shock front. According to the Snell-Descartes law, larger refraction will occur at larger shock front angles. The electron beam centroid then follows the direction of the laser, leading to a vertical offaxis deflection when $\alpha$ is nonzero. This trend can be seen in Fig. 4(a). Using measured density profiles and shock front
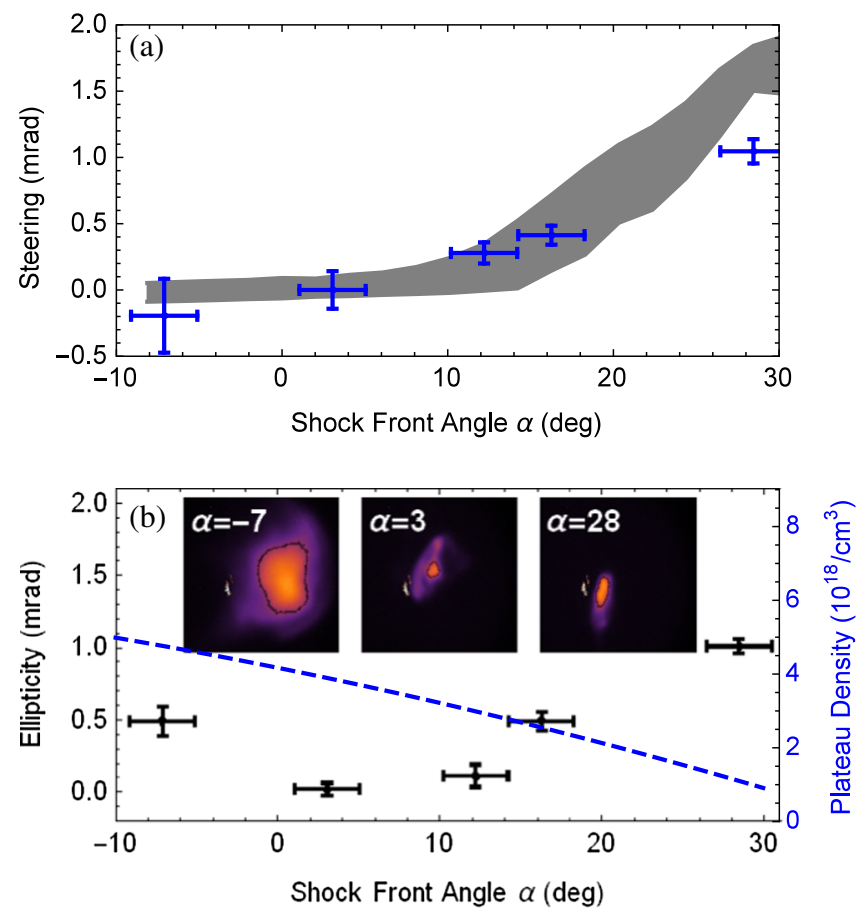

FIG. 4. (a) Steering versus shock front angle. Experimental data on electron beam steering is shown as the blue points while the gray area displays laser refraction calculated from measured density profiles. (b) Ellipticity (black points) and $n_{2}$ (dashed line) versus shock front angle. Insets show electron beam spatial profiles for different $\alpha$. angles, the expected total refraction of the laser as it propagates through the gas was calculated and is displayed in the gray area. The laser refraction agrees well with the experimentally measured electron beam deviation. Therefore, off-axis propagation can be mitigated by reducing the shock front angle.

Laser refraction also influences the beam ellipticity, defined as the difference between vertical and horizontal divergence, as shown in Fig. 4(b). Due to laser steering, the electron beam position becomes displaced from the laser axis, inducing betatron oscillations. The larger the laser refraction, the more the electrons are displaced and the larger the betatron amplitude. As this effect occurs only along the shock axis, it results in elliptical beams in the vertical direction.

In the experiments, the laser polarization was vertical, and elliptical electron beams can also result through interaction of the electrons with the laser. The degree of ellipticity increases with the ratio $c \tau / \lambda_{p, 2} \propto \sqrt{n_{2}}$, where $\tau$ is the duration of the laser pulse [31]. When $n_{2}=5.0 \times$ $10^{18} \mathrm{~cm}^{-3}, \lambda_{p}$ is comparable to the laser duration, suggesting that at higher plateau densities, electron beam ellipticity might be attributed to laser effects. However, at lower densities, $\lambda_{p}$ is much longer than the laser duration, and the laser electric field has a reduced influence. In this regime, the primary cause of ellipticity is the tilted shock front, and we find that ellipticity was minimized when the shock front was nearly perpendicular to the laser propagation direction.

The absolute energy spread $\Delta E$ was reduced by changing the high density region length $L_{\text {high }}$ where $L_{\text {high }}$ is defined as the distance from where the density first exceeds $10^{18} \mathrm{~cm}^{-3}$ to the density peak [see Fig. 2(a)]. Decreasing the backing pressure or the vertical distance between the jet assembly and the laser caused $L_{\text {high }}$ to decrease. It was observed that a decrease in $L_{\text {high }}$ reduced $\Delta E$ as shown in Fig. 5. The backing pressure and jet-laser height also affected $n_{1}$, but a comparison of multiple scans showed that $\Delta E$ was dominated by $L_{\text {high }}$ rather than $n_{1}$ as detailed in

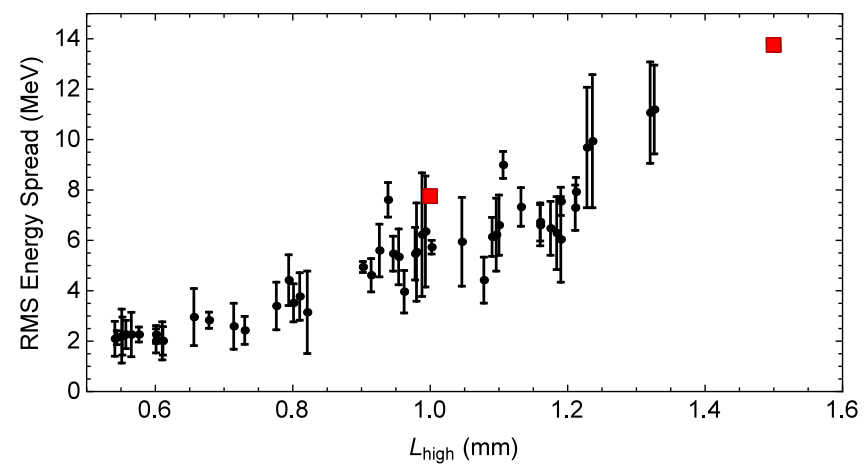

FIG. 5. Energy spread of electron beams against the width of the high density region. Black circles represent experimental data, and red squares represent simulation results. 
[32]. By reducing $L_{\mathrm{high}}$, the absolute energy spread was minimized to $\Delta E=2 \mathrm{MeV}$ RMS for energies on the order of $100 \mathrm{MeV}$, allowing percent-level energy spreads to be attained. In principle, downramp injection can produce electron beams with smaller energy spreads than we attain here via precise control of the plasma density profile [21], but limitations imposed by the implemented jet and blade configuration prevented us from reaching this limit.

At constant backing pressure and jet-laser height, when the blade coverage was changed, $\Delta E$ remained fairly constant as shown in Fig. 3c. This can be explained from the density profile measurements which showed that $L_{\text {high }}$ was only weakly dependent on blade coverage. Therefore, $\Delta E$, which is correlated to $L_{\text {high }}$, is not coupled to the final energy $W$, which is correlated to $L_{\text {acc }}$, suggesting that $\Delta E$ was conserved during the acceleration process. This observation demonstrates that the electron beam energy and energy spread can be independently tuned.

\section{SIMULATIONS}

The axisymmetric processes were modeled using the spectral, quasi-3D particle-in-cell code FBPIC [33], providing more insight into the injection process. A key feature of the density profile is the up-ramp, which governs the laser propagation and therefore wake properties at the shock when electrons are injected. The effect of the up-ramp was examined by simulating two density profiles. The upramp regions matched two experimental profiles while the downramp regions were made identical to decouple upramp and downramp effects on the electron beam. The two profiles are shown in Fig. 6(a) where the wide profile has a $1.5 \mathrm{~mm}$ up-ramp length and the narrow has a $1.0 \mathrm{~mm}$ upramp length.

Simulations show that the laser self-focuses until it reaches the density downramp, increasing the peak longitudinal accelerating field $E_{z}$ as seen in Fig. 6(a). The wide
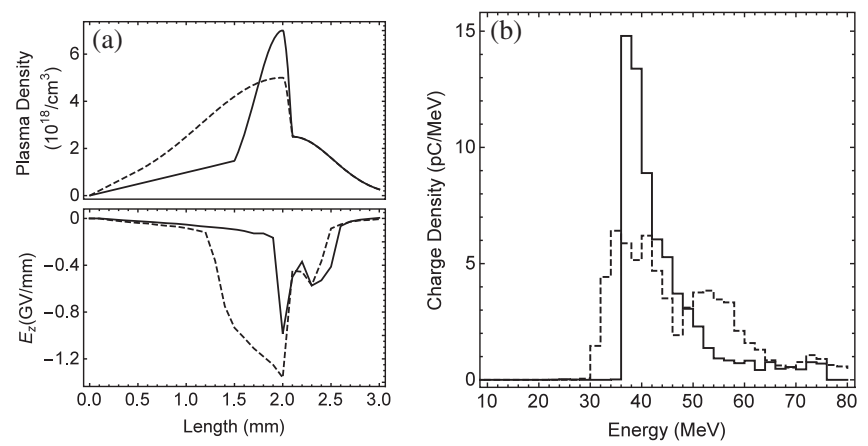

FIG. 6. (a) Particle-in-cell simulations showing the plasma density profile and the peak longitudinal $E_{z}$ field at the back of the plasma bubble as a function of propagation distance for a wide up-ramp (dashed) and narrow up-ramp (solid) density profile. (b) Electron energy spectra at the end of the downramp for the wide up-ramp (dashed) and narrow up-ramp (solid) density profile. and narrow up-ramp profiles induced differing amounts of self-focusing. Most noticeably, at the start of the downramp when the electrons are first being injected, the wide up-ramp profile produced a larger amplitude $E_{z}$ field while the $E_{z}$ fields were similar along the rest of the profile. Figure 6(c) shows the final energy spectra of the electrons injected in the two different profiles. The wide up-ramp profile produced electron beams with an energy spread of $16 \mathrm{MeV}$ while the narrow up-ramp profile beams had a spread of $9 \mathrm{MeV}$ which is consistent with the experimental data shown in Fig. 5. The wide up-ramp profile injected more electrons with energies greater than $50 \mathrm{MeV}$. These electrons were injected from $z=2.0$ to $z=2.1 \mathrm{~mm}$ where the wide up-ramp had a larger $E_{z}$ amplitude. Therefore, the larger range in $E_{z}$ values for the wide up-ramp induced a larger energy spread by accelerating the initially injected electrons to higher energies. These results explain the correlation between energy spread and up-ramp width.

\section{CONCLUSIONS}

In conclusion, stable and controllable high-quality electron beams have been experimentally produced in a shockinduced density downramp LPA through density and shock front angle tailoring while maintaining the laser focal location. Using simple models, the relationships between the plasma profile and the resulting electron beams were explained. The highest quality beams, specifically concerning ellipticity and on-axis steering, were produced with a shock front perpendicular to the laser propagation direction. In addition, the up-ramp length allowed energy spread control, with the shortest length resulting in an absolute energy spread of $2 \mathrm{MeV}$, while maintaining independently-tunable energies in the $30-180 \mathrm{MeV}$ range. The quality and stability of these beams are suitable for LPA applications. Particularly, the achieved $3.5 \mathrm{pC} / \mathrm{MeV}$ spectral charge density at $62 \mathrm{MeV}$ make the beams attractive for free-electron lasing [34]. Achieving such charge densities at few-percent energy spread reduces emittance degradation from collective effects such as coherent synchrotron radiation and space charge [34]. Similarly, stable few-percent energy spread beams are appropriate to enable Thomson scattering sources of $\mathrm{MeV}$ photons [12]. In addition, the achieved percent-level energy spread allows for efficient coupling in a multistage accelerator since the electron bunch duration, as determined by its energy spread, must fit within the accelerating phase of the wake.

\section{ACKNOWLEDGMENTS}

This work is supported by the U.S. DOE under Contract No. DE-AC02-05CH11231, by the U.S. DOE National Nuclear Security Administration, Defense Nuclear Nonproliferation R\&D (NA22), and by the National Science Foundation under Grant No. PHY-1415596. 
[1] T. Tajima and J. M. Dawson, Phys. Rev. Lett. 43, 267 (1979).

[2] E. Esarey, C. B. Schroeder, and W. P. Leemans, Rev. Mod. Phys. 81, 1229 (2009).

[3] W. P. Leemans, A. J. Gonsalves, H.-S. Mao, K. Nakamura, C. Benedetti, C. B. Schroeder, C Toth, J. Daniels, D. E. Mittelberger, S. S. Bulanov, J.-L. Vay, C. G. R. Geddes, and E. Esarey, Phys. Rev. Lett. 113, 245002 (2014).

[4] C. G. R. Geddes, C. Toth, J. van Tilborg, E. Esarey, C. B. Schroeder, D. Bruhwiler, C. Nieter, J. Cary, and W. P. Leemans, Nature (London) 431, 538 (2004).

[5] J. Faure, Y. Glinec, A. Pukhov, S. Kiselev, S. Gordienko, E. Lefebvre, J.-P. Rousseau, F. Burgy, and V. Malka, Nature (London) 431, 541 (2004).

[6] C. B. Schroeder, E. Esarey, W. P. Leemans, J. van Tilborg, F. J. Grner, A. R. Maier, Y. Ding, and Z. Huang, in Proceedings of 2013 Free-Electron Laser Conference, New York (AIP Publishing, Manhattan, New York, 2013), p. MOPSO69.

[7] A. R. Maier, A. Meseck, S. Reiche, C. B. Schroeder, T. Seggebrock, and F. Gruner, Phys. Rev. X 2, 031019 (2012).

[8] S. Chen, N. D. Powers, I. Ghebregziabher, C. M. Maharjan, C. Liu, G. Golovin, S. Banerjee, J. Zhang, N. Cunningham, A. Moorti, S. Clarke, S. Pozzi, and D. P. Umstadter, Phys. Rev. Lett. 110, 155003 (2013).

[9] H.-E. Tsai, X. Wang, J. M. Shaw, Z. Li, A. V. Arefiev, X. Xhang, R. Zgadzaj, W. Henderson, V. Khudik, G. Shvets, and M. C. Downer, Phys. Plasmas 22, 023106 (2015).

[10] R. Neutze, R. Wouts, D. van der Spoel, E. Weckert, and J. Hajdu, Nature (London) 406, 752 (2000).

[11] A. Cummings, G. O'Sullivan, P. Dunne, E. Sokell, N. Murphy, J. White, P. Hayden, P. Sheridan, M. Lysaght, and F. O'Reilly, J. Phys. D 39, 73 (2006).

[12] C. G. R. Geddes, S. Rykovanov, N. H. Matlis, and W. P. Leemans, Nucl. Instrum. Methods Phys. Res., Sect. B 350, 116 (2015).

[13] A. Pukhov and J. Meyer-ter-Vehn, Appl. Phys. B 74, 355 (2002).

[14] V. Malka, S. Fritzler, E. Lefebvre, M.-M. Aleonard, F. Burgy, J.-P. Chambaret, J.-F. Chemin, K. Krushelnick, G. Malka, S. P. D. Mangles et al., Science 298, 1596 (2002).

[15] M. Chen, Z.-M. Sheng, Y.-Y. Ma, and J. Zhang, J. Appl. Phys. 99, 056109 (2006).

[16] C. McGuffey, A. G. R. Thomas, W. Schumaker, T. Matsuoka, V. Chvykov, F. J. Dollar, G. Kalintchenko, V. Yanovsky, A. Maksimchuk, K. Krushelnick et al., Phys. Rev. Lett. 104, 025004 (2010).

[17] S. P. D. Mangles, C. D. Murphy, Z. Najmudin, A. G. R. Thomas, J. L. Collier, A. E. Dangor, E. J. Divall, P. S. Foster, J. G. Gallacher, C. J. Hooker et al., Nature (London) 431, 535 (2004).
[18] A. Pak, K. A. Marsh, S. F. Martins, W. Lu, W. B. Mori, and C. Joshi, Phys. Rev. Lett. 104, 025003 (2010).

[19] E. Esarey, R. F. Hubbard, W. P. Leemans, A. Ting, and P. Sprangle, Phys. Rev. Lett. 79, 2682 (1997).

[20] J. Faure, C. Rechatin, A. Norlin, A. Lifschitz, Y. Glinec, and V. Malka, Nature (London) 444, 737 (2006).

[21] C. G. R. Geddes, K. Nakamura, G. R. Plateau, C. Toth, E. Cormier-Michel, E. Esarey, C. B. Schroeder, J. R. Cary, and W. P. Leemans, Phys. Rev. Lett. 100, 215004 (2008).

[22] A. J. Gonsalves, K. Nakamura, C. Lin, D. Panasenko, S. Shiraishi, T. Sokollik, C. Benedetti, C. B. Schroeder, C. G. R. Geddes, J. van Tilborg et al., Nat. Phys. 7, 862 (2011).

[23] S. Bulanov, N. Naumova, F. Pegoraro, and J. Sakai, Phys. Rev. E 58, R5257 (1998).

[24] K. Schmid, A. Buck, C. M. S. Sears, J. M. Mikhailova, R. Tautz, D. Herrmann, M. Geissler, F. Krausz, and L. Veisz, Phys. Rev. ST Accel. Beams 13, 091301 (2010).

[25] A. Buck, J. Wenz, J. Xu, K. Khrennikov, K. Schmid, M. Heigoldt, J. M. Mikhailova, M. Geissler, B. Shen, F. Krausz, S. Karsch, and L. Veisz, Phys. Rev. Lett. 110, 185006 (2013).

[26] E. Guillaume, A. Dopp, C. Thaury, K. T. Phuoc, A. Lifschitz, G. Grittani, J.-P. Goddet, A. Tafzi, S. W. Chou, L. Veisz, and V. Malka, Phys. Rev. Lett. 115, 155002 (2015).

[27] G. R. Plateau, N. H. Matlis, C. G. R. Geddes, A. J. Gonsalves, S. Shiraishi, C. Lin, R. A. van Mourik, and W. P. Leemans, Rev. Sci. Instrum. 81, 033108 (2010).

[28] H. G. Weller, G. Tabor, H. Jasak, and C. Fureby, Comput. Phys. 12, 620 (1998).

[29] H.-S. Mao, K. K. Swanson, S. K. Barber, H.-E. Tsai, S. Steinke, J. van Tilborg, C. G. R. Geddes, and W. P. Leemans (to be published).

[30] M. Reiser, Theory and Design of Charged Particle Beams (Wiley, New York, 2008).

[31] S. P. D. Mangles, A. G. R. Thomas, M. C. Kaluza, O. Lundh, F. Lindau, A. Persson, F. S. Tsung, Z. Najmudin, W. B. Mori, C.-G. Wahlstrom, and K. Krushelnick, Phys. Rev. Lett. 96, 215001 (2006).

[32] H.-E. Tsai, K. Swanson, S. Barber, R. Lehe, H.-S. Mao, S. Steinke, J. van Tilborg, C. G. R. Geddes, and W. P. Leemans, in Proceedings of the Advanced Accelerator Concepts, 2016 (to be published).

[33] J.-L. Vay, D. P. Grote, R. H. Cohen, and A. Friedman, Comput. Sci. Discovery 5, 014019 (2012).

[34] J. van Tilborg, S. K. Barber, F. Isono, C. B. Schroeder, E. Esarey, and W. P. Leemans, Advanced Accelerator Concepts Workshop, 2016 (unpublished). 